trée dans le vide. On obtient finalement non pas un corps cristallin, mais un sirop très pur qui ressemble à la glycérine.

De même, le lactate de calcium peut être employé comme intermédiaire pour la fabrication de l'acide lactique lui-même. A cet effet, le sel très pur est dissous dans l'eau chaude et la solution est acidifiée avec de l'acide sulfurique concentré ou légèrement dilué. Après passage au filtre-presse, on obtient une solution de l'acide généralement colorée par suite de la présence d'impuretés métalliques, Ces impuretés peuvent généralement être éliminées par traitement au moyen du ferrocyanure de potassium ou de tout autre réactif et au moyen de charbon.

\title{
A PROPOS DE L'EMPLOI DE LA CASÉINE DANS LA FABRICATION DES MATIËRS PLASTIQUES
}

Nous avons publié dans notre numéro de septembre-octobre 1937 une étude que nous avons voulu très succincte sur la fabrication des matières plastiques à base de caséine et nous avons en particulier rappelé quelques faits sur l'historique de cette industrie.

Un de nos fidèles lecteurs, M. Marc FouAssIen, nous écrit très aimablement pour nous informer, qu'à son avis, nous n'avons pas assez insisté sur l'importance essentielle, pour l'avenir de cette industrie, d'une découverte faite par un savant français, M. TrILlat, et nous communique quelques renseignements.

Devant l'intérêt que présente ce point d'histoire, nous avons demandá à notre collaborateur, M. GÉnIN, de réunir les informations qu'il a pu recueillir sur l'origine de la fabrication des matières plastiques à base de caséine, afin de compléter sa précédente note.

Il semble que c'est par un chimiste dénommé F. E. CHILds (Brevet allemand 32.293 ) que la première mention a été faite de l'emploi de la caséine du lait pour la fabrication des matières plastiques. Dans le procédé décrit par cet auteur vers 1884, on utilisait le coagulum obtenu en laissant sûrir le lait; ce coagulum était égoutté, découpé, lavé à l'eau bouillante, puis malaxé après introduction des différentes substances destinées à le rendre plus dur; finalement on le laminait ou on le moulait.

On trouve ensuite quelques années plus tard un procédé analogue décrit par O. Schoenfeld (Brevet allemand 85.886 de 1893) d'après lequel on obtenait des masses plus ou moins dures en utilisant un mélange de easéine, de matière colorante et d'oxyde de zinc, puis e'est le brevet 118.952 de C. JUNG, A. Brecher et A. KITten dans lequel on partait comme matière première de caséinate alcalin qui était, après séchage, mólangé avec des huiles sulfurées, ainsi qu'avec du caoutchouc, des résines et des matières colorantes (Brevet 
allemand 118,952). C'est enfin, une importante découverte de A. Spitteler décrite dans le brevet allemand 115.681 qui permettait par une élimination des cendres de la caséine d'obtenir un produit translucide alors que toutes les substances préparées jusqu'alors étaient opaques. Ce procédé dans lequel on utilisait comme agent déminéralisant une solution d'alcali-caustique fut quelque peu modifié par les "Vereinigten Gummiwaren-Fabriken NarburgWien" (Brevet allemand 141.309) par remplacement de l'alcali caustique par des solutions de carbonates alcalins.

Mais, quel que soit le procédé employé, la substance obtenue n'était pas insoluble et c'est à un savant français, M. TrmLAT, qu'on doit indiscutablement la découverte fondamentale que possède la formaldéhyde d'insolubiliser les matières albuminoïdes (Compte rendu de l'Académie des Sciences, t. I, 1892, p. 1280). En 1893, ce savant put préparer dans les Laboratoires de la Maison Sordes et Huillard, à Suresnes, des plaques de gélatine et de caséine durcies au formol; l'application se borna tout d'abord à l'insolubilisation des objets en gélatine, notamment des paillettes dont l'industrie prit à cette époque un développement important.

C'est alors que l'observation de M. Trillat fut discutée par différents chimistes allemands; en particulier, dans un brevet allemand 99.505 de la Chemische Fabrik a.A. vorm.E. Schering, il est indiqué que le procédé décrit par TrILLAT ne permettait pas à coup sûr d'insolubiliser par exemple le blanc d'œuf ou l'albumine. On dit en effet dans ce brevet que cette insolubilisation ne s'obtient que par dessiceation complète du blane d'œuf qui doit être alors traité par le formol. Plus tard, la même Société (Brevet allemand 107.637 et Brevet Français 236.178 du 10 février 1894) prit un brevet pour le durcissement de la gélatine, de la colle, de la caséine par la formaldéhyde, par insolubilisation obtenue en évaporant les solutions contenant le formol.

Les brevets sur la préparation de masses plus ou moins dures obtenues par insolubilisation de caséine deviennent alors plus nombreux. Signalons en particulier le Brevet allemand 123.815 de $\mathrm{J}$. Mensik. Cependant, tous les procédés décrits avaient l'inconvénient de donner des produits qui manquaient de plasticité et dont le moulage était par conséquent difficile. C'est à SPITTELer qu'on doit à nouveau une amélioration du procédé et la découverte d'un mode de traitement qui devait donner naissance à une industrie très florissante, celle de la "Galalith ", qu'il ne faut donc pas confondre, comme nous le fait remarquer M. FouASSIER, avee la simple caséine formolée.

Les améliorations apportées à cette industrie par SPITTELER sont en réalité dues à la collaboration de deux chimistes, A. SpitTe- 
LeR et W. Krische. A l'époque, Krische, directeur d'une grande imprimerie de Hanovre, recherchait la possibilité d'obtenir des ard jises blanches, lavables, incassables, sur lesquelles on pouvait ézrire au crayon et qui pouvaient ensuite être lavées. SPITteler, de son côté, essayait d'améliorer la production de masses plastiques en partant de caséine et c'est en mettant en commun leurs efforts, que ces deux auteurs parvinrent à entreprendre la fabrication de ce qu'on appelle aujourd'hui la "galalith".

Cet historique de la question est aujourd'hui bien peu connu de tous ceux qui utilisent et fabriquent la galalith, mais presque tous ignorent lis travaux de M. TRILLAT et se contentent évidemment de rappeler dæns leurs études les recherches des savants allem ^nds qui aboutirent à la dścouverte de la "galalith ".

\section{BIBLIOGRAPHIE ANALYTIQLE}

\section{LES LIVRES}

\section{LAXA (O.). - Chemie maléka a mlécnych vyrobku, II. dil, Mlec-} né vyrobky (La chimie du lait et des produits laitiers. Vol. II. L ss produits laitiers). Publication du Ministère de l'Agriculture de la République Tchésoslovaque, $n^{\circ} 92$, Prague 1936. 1 volume broché, 327 pages, 54 photogra phies, 188 tableaux. Prix . broché 45 couronnes tchèques.

Le nouveau livre de LAXA termine son ouvrage sur la chimie du lait et des produit-laitiers et fait suite au volume précédent traitant de la chimie du lait. Il est divisé en 5 chapitres principaux dont le premier traite des produits semblables au lait (lait stérilisé, lait pour enfants et laits transformés, lait écrémé, babeurré, petit-lait, lait caillé et fermenté). Le chapitre II, traite des conserves du lait et de Ia farine lactée ; le chapitre III, de la crème fraîche, de la crème en poudre et de la erème glacée; le chapitre IV, du beurre et le chapitre V, des fromages. A ces chapitres, se joignent encore les articles sur la caséine et sur la boue d'écrémeuses. L'index bibliographique et la table des matières terminent l'ouvrage.

Le contenu des chapitres cités montre que le livre contient tout ce qui concerne les produits laitiers. Tous les produits sont cités par des définitions justes, et l'A. y traite de leur composition chimique et des changements auxquels les différents éléments sont soumis. En même temps, il cite minutieusement les causes de cee changements. L'A. porte son attention sur la valeur biologique et nutritive aussi bien que sur la digestibilité des différents produits laitiers, et ce livre est une source d'information pour celui qui veut juger les produits laitiers au point de vue de la nutrition.

Le livre apporte une grande quantité de matériaux analytiques, non seulement ceux puisés dans la littérature universelle, mais aussi les résultats 